\section{Prevalencia de sensibilización al látex mediante prueba cutánea (prick test) en pacientes con malformaciones genitourinarias con más de tres intervenciones quirúrgicas}

Ana Paola Macías-Robles, ${ }^{1}$ Ana Rocío Morán-Mendoza ${ }^{2}$

\section{Resumen}

INTRODUCCIÓN: la alergia al látex tiene alta prevalencia en grupos de riesgo conocidos, especialmente en pacientes con espina bífida, malformaciones urinarias y ortopédicas con múltiples cirugías. En México no se cuenta con suficientes estudios que reporten la prevalencia y factores de riesgo asociados.

OBJETIVO: determinar la prevalencia de sensibilización al látex mediante prueba de prick con extracto de látex en pacientes con malformaciones genitourinarias y más de tres cirugías en la Unidad Médica de Alta Especialidad de Pediatría del Centro Médico Nacional de Occidente, así como los factores asociados.

RESULTADOS: la prevalencia encontrada fue de $30.7 \%$ y los factores de riesgo asociados: atopia personal $(p=0.047)$, antecedente de reacción previa con productos con látex $(p=0.003)$, específicamente con globos $(p=0.000)$ y guantes $(p=0.002)$. No hubo asociación entre el número de cirugías e intervenciones quirúrgicas a edades tempranas, tampoco con concentraciones elevadas de IgE sérica total. Tuvieron reacción cruzada a frutas-látex $25 \%$ de los pacientes, los alimentos asociados fueron: aguacate, papaya, fresa y kiwi.

CONCLUSIÓN: la prevalencia de sensibilización al látex es alta en los grupos de riesgo, sobre todo con antecedente de atopia, por lo que es importante que el personal de salud identifique a estos pacientes a fin de implementar oportunamente las medidas de prevención primaria y secundaria; evitar efectos severos potencialmente mortales, como la anafilaxia, para disminuir la morbilidad y la mortalidad y los costos sanitarios.

PALABRAS CLAVE: sensibilización a látex, alergia, prueba de PRICK, atopia, cirugías.

\begin{abstract}
${ }^{1}$ Servicio de Alergia e Inmunología Clínica, Hospital de Especialidades, Centro Médico Nacional Siglo XXI, Instituto Mexicano del Seguro Social, Ciudad de México, México.

${ }^{2}$ Servicio de Alergia e Inmunología Clínica, Hospital de Pediatría, Centro Médico Nacional de Occidente, Instituto Mexicano del Seguro Social, Guadalajara, Jalisco, México.
\end{abstract}

Recibido: 2 de octubre 2015

Aceptado: 22 de febrero 2016

Correspondencia

Ana Paola Macías-Robles

Santa Elena 2317, Guadalajara, Jalisco.

paola_maro@hotmail.com

Este artículo debe citarse como

Macías-Robles AP, Morán-Mendoza AR. Prevalencia de sensibilización al látex mediante prueba cutánea (prick test) en pacientes con malformaciones genitourinarias con más de tres intervenciones quirúrgicas. Rev Alerg Méx. 2016 abr-jun;63(2):154-162. 


\section{Latex sensitization prevalence through prick test in patients with genitourinary malformations and more than 3 surgeries}

\author{
Ana Paola Macías-Robles, ${ }^{1}$ Ana Rocío Morán-Mendoza²
}

\begin{abstract}
BACKGROUND: Latex allergy is a public health issue. It presents elevated prevalence in known risk groups, especially in those patients with spine bifida condition, urinal malformation and for those with orthopedic problems - multiple surgeries. Health Services in Mexico do not have the enough studies about prevalence and risk associated to latex allergy.
\end{abstract}

OBJECTIVE: Determine latex sensitization prevalence through PRICK test in patients with genitourinary malformations and more than 3 surgeries in UMAE pediatric CMNO unit, considering too related factors.

MATERIAL AND METHOD: An analytical and descriptive crosssectional study was performed, which included men and women from 1 to 16 years with genitourinary malformations and more than three surgeries. A survey to know the risk factors associated and prick test was performed with latex extract, with positive and negative control. Serum levels of total IgE and eosinophils were measured in peripheral blood.

RESULTS: The study exposed prevalence of $30.72 \%$. Related to associated factors as follows: atopy $(p=0.047)$, previous antecedent reaction to latex products $(p=0.003)$ specific for balloons $(p=0.000)$ and gloves $(p=0.002)$. There was not association related to amount of surgeries and surgical interventions on early age, either for high levels of total serum IgE.

CONCLUSION: Prevalence to latex sensitization is high in risk groups. Especially in those with atopy thereby is important for health personal to identify these patients in order to implement on time the preventive primary/secondary measures. With these actions potential mortal risks like anaphylaxis will be avoided. This will decrease sanitary costs and mortality.

KEYWORDS: Latex sensitization, allergy, prick test, atopy, surgeries.

\footnotetext{
${ }^{1}$ Servicio de Alergia e Inmunología Clínica, Hospital de Especialidades, Centro Médico Nacional Siglo XXI, Instituto Mexicano del Seguro Social, Ciudad de México, México. ${ }^{2}$ Servicio de Alergia e Inmunología Clínica, Hospital de Pediatría, Centro Médico Nacional de Occidente, Instituto Mexicano del Seguro Social, Guadalajara, Jalisco, México.

\section{Correspondence}

Ana Paola Macías-Robles

Santa Elena 2317, Guadalajara, Jalisco.

paola_maro@hotmail.com
}

\section{ANTECEDENTES}

El látex se obtiene a partir de la savia lechosa del árbol del caucho Hevea brasiliensis; existen más de 4000 productos que contienen látex, utilizados en el hogar y en el medio hospitalario. ${ }^{1}$ La Unión Internacional de Sociedades Inmunológicas (IUIS) reconoce 15 alergenos del látex (Hev b1-Hev b 15). Los alergenos al látex de mayor importancia clínica son Hev b 1 y Hev 
b 3 en pacientes pediátricos con espina bífida, alteraciones genitourinarias y $\mathrm{Hev}$ b5, Hev b 6 y Hev b 13 en trabajadores de la salud. ${ }^{2}$

La alergia al látex constituye hoy en día un problema médico común. La primera descripción de hipersensibilidad inmediata por látex se realizó en Alemania en $1927,{ }^{3}$ la siguiente apareció en $1979,{ }^{4}$ y fue a finales del decenio de 1980 y coincidiendo con las recomendaciones internacionales para evitar el contagio del VIH y otras enfermedades infecciosas que implicaron un gran aumento en la utilización de guantes, cuando la alergia al látex tuvo un incremento importante. Si bien la alergia a látex ha decaído en Estados Unidos en los últimos años, de 150,000 a 1'000,000 de trabajadores de la salud, cerca de 15 millones de personas en el mundo aún sufren alergia al látex. ${ }^{5}$

La alergia al látex es un problema de salud pública en pacientes de riesgo. Aunque en la actualidad la alergia al látex se reconoce como poco frecuente 1 a $2 \%$ de la población en general, aún existen grupos de riesgo bien diferenciados.

Entre los profesionales de la salud, el riesgo de alergia al látex varía de 2.5 a $25 \%$ según los autores, variabilidad que puede deberse a la población estudiada, al país donde se efectuó y al método aplicado para diagnosticar la alergia a látex. El riesgo de alergia al látex entre otros profesionales varía en función de la frecuencia e intensidad de la exposición; la probabilidad es mayor entre los que trabajan en los quirófanos, donde la exposición al látex es muy superior $(10.7 \%)$ frente al $2 \%$ registrado entre los que ejercen en otras áreas del hospital. ${ }^{6}$

Otro grupo importante son los niños con espina bífida o con anomalías urogenitales que requieren múltiples intervenciones quirúrgicas y colocación de sondas, que se encuentran a permanencia o sondeo intermitente por lo que el riesgo de alergia al látex es mayor que el de la población en general. La prevalencia en este grupo varía entre $32.6 \%$ en estudios que utilizan pruebas cutáneas y entre $34-72 \%$ en los basados en pruebas serológicas. Los principales factores de riesgo son los antecedentes atópicos y el número de intervenciones quirúrgicas. El riesgo es mayor cuando hay intervenciones quirúrgicas en los primeros días de vida. ${ }^{7}$

Otros grupos de riesgo son los pacientes alérgicos a alimentos de origen vegetal o con síndrome látex-frutas, que se caracterizan por la reacción cruzada entre los alergenos del látex con los de algunas frutas, sobre todo aguacate, kiwi o castaña. Aproximadamente la mitad de los pacientes alérgicos al látex muestran alergia a determinados alimentos. A su vez, la mitad de estas reacciones a alimentos se manifiestan como anafilaxia sistémica, repartiéndose la otra mitad entre "síndrome de alergia oral", urticaria o angioedema. Los alimentos más comúnmente implicados son: plátano $(28 \%)$, aguacate $(28 \%)$, castaña $(24 \%)$ y kiwi $(20 \%)$; la proporción varía según el consumo relativo de dichos alimentos en cada zona. ${ }^{8}$

El objetivo de este estudio fue: determinar la prevalencia de sensibilización al látex mediante prueba cutánea (Prick test) en pacientes con malformaciones genitourinarias con tres o más intervenciones quirúrgicas en el Hospital de Pediatría del Centro Médico Nacional de Occidente y los factores de riesgo asociados.

\section{MATERIAL Y MÉTODO}

Estudio transversal analítico y descriptivo que incluyó hombres y mujeres de 1 a 16 años, derivados de la consulta de Urología Pediátrica del Hospital de Pediatría del Centro Médico Nacional de Occidente, con malformaciones genitourinarias y más de tres cirugías, al servicio 
de Alergia e Inmunología Clínica Pediátrica de septiembre de 2014 a enero de 2015. Se aplicó una encuesta para conocer los factores de riesgo asociados y se aplicó una prueba cutánea por punción con extracto de látex, control positivo y negativo, se midieron las concentraciones séricas de IgE total y eosinófilos en sangre periférica.

La interpretación de las pruebas cutáneas se efectuó de acuerdo con las indicaciones internacionales propuestas por la Academia Europea de Alergia e Inmunología Clínica (EAACI). Se excluyeron del estudio los pacientes que hubieran ingerido antihistamínicos 10 días previos a la realización de la prueba, los que se aplicaron esteroide tópico seis semanas previas al estudio, y a los pacientes con inmunodeficiencia concomitante, con dermatosis generalizada y los menores de un año de edad.

Se consideraron pacientes con resultado positivo (sensibilización a látex) los que presentaron al reactivo de extracto de látex un habón de 3 mm o mayor en comparación con el control negativo. Se utilizó extracto Allergomex látex (Abercomex).

Las concentraciones de IgE sérica total se midieron con el método de nefelotomía con reactivos látex N IgE Reagent Siemens y las de eosinófilos con el equipo Adria 120 Hematology System; se procesaron en el laboratorio del Hospital de Pediatría del Centro Médico Nacional de Occidente.

\section{Consideraciones éticas}

El protocolo se apega a las normas internacionales para la investigación médica en seres humanos promulgados en la Declaración de Helsinki de la Asociación Médica Mundial, enmendada en la 59a Asamblea celebrada en Seúl, Corea del Sur, en octubre de 2008. Cumple con la normatividad relativa a investigación

\section{Análisis estadístico}

Para las variables cualitativas y cuantitativas se realizaron frecuencias y proporciones. Se utilizó una base de datos en Excel, el análisis de los datos se reunió en el paquete estadístico SPSS versión 21.0 para Windows

Los resultados se expresaron en porcentajes de frecuencia y en números absolutos. Mediante análisis de $X^{2}$ y correlación se evaluó la relación entre los fenotipos y la sensibilización. El valor de $p<0.05$ se consideró significativo. Se compararon los factores de riesgo asociados con los pacientes con prueba de prick positiva con la prueba $U$ de Mann-Whitney de muestras independientes.

\section{RESULTADOS}

\section{Características de los pacientes}

Se evaluaron 52 pacientes de los que 35 correspondieron al género masculino $(67 \%)$ y 17 al femenino (33\%); la media de edad fue de 6 años (límites 1 y 16 años). De los pacientes estudiados 16 de 52 (31\%) tuvieron prueba de prick cutánea positiva con extracto de látex.

En el Cuadro 1 se presentan las características sociodemográficas de los 52 pacientes estudiados.

\section{Resultados de pacientes con prueba cutánea (prick) positiva al látex}

En los pacientes con sensibilización al látex mediante prueba cutánea se encontró:

Género: 13 pacientes (81\%) de sexo masculino y $3(19 \%)$ del femenino. Se reportó una mediana de edad de 11 años (mínima 5, máxima 15 años).

Malformaciones genitourinarias: un paciente tuvo alteración renal, otro alteración genital, y 14 alteración vesical y uretral. 
Cuadro 1. Características sociodemográficas de los pacientes

\begin{tabular}{lc}
\hline Variable & $\mathbf{n}$ \\
Sexo & \\
- Femenino: $\mathrm{n}(\%)$ & $17(33 \%)$ \\
- Masculino: $\mathrm{n}(\%)$ & $35(67 \%)$ \\
Edad años, med (rango) & \\
- $1-4 \mathrm{n}(\%)$ & $7(13 \%)$ \\
- $5-9 \mathrm{n}(\%)$ & $17(33 \%)$ \\
- $10-13 \mathrm{n}(\%)$ & $17(33 \%)$ \\
- $\geq 14 \mathrm{n}(\%)$ & $11(21 \%)$ \\
Tipo de alteración genitourinaria & \\
- Renal n (\%) & $5(10 \%)$ \\
- Vesical n (\%) & $44(84 \%)$ \\
- Genital n (\%) & $3(6 \%)$ \\
No. de intervenciones quirúrgicas, med (rango) \\
- $4-8 \mathrm{n}(\%)$ \\
- 9-13 n (\%) \\
- $\geq 14 \mathrm{n}(\%)$ & $5(10 \%)$ \\
Edad de Primera Intervención Quirúrgica & $44(84 \%)$ \\
$\leq 30$ días & $3(6 \%)$ \\
$>30$ días & $29(56 \%)$ \\
\end{tabular}

\section{Intervenciones quirúrgicas}

La media de intervenciones quirúrgicas por paciente fue de 6.5 cirugías. No se encontró asociación estadísticamente significativa y el número elevado de cirugías $(\mathrm{p}=0.481)$ tampoco lo fue la edad menor de 30 días a la primera intervención quirúrgica para sensibilización a látex $(p=0.209)$.

Con antecedente de reacción alérgica a medicamentos se reportaron 6 pacientes (37.5\%), los medicamentos reportados fueron: penicilina (dos pacientes), trimetoprima-sulfametoxazol (un paciente), eritromicina (un paciente), ciprofloxacina (un paciente), oxibutinina (un paciente). Sin asociación entre alergia a fármacos y prick positivo a látex $(p=0.252)$.

\section{Antecedente personal de atopia.}

Se encontró asociación con el antecedente personal de atopia $(\mathrm{p}=0.047)$, y prick positivo. El antecedente de cuadros previos de urticaria y angioedema $(p=0.043)$ y el antecedente de anafilaxia $(p=0.008)$ sí tuvieron influencia; no así la relación con el antecedente de dermatitis atópica $(p=0.657)$, ni el antecedente de rinitis o asma ( $\mathrm{p}=0.234)$ (Cuadro 2).

\section{Reacción cruzada fruta-látex}

El 25\% de los pacientes tuvo reacción cruzada fruta- látex ( $p=0.043)$, que se identificó mediante interrogatorio dirigido. Los alimentos relacionados fueron: aguacate, papaya, pera y kiwi (Cuadro 3).

\section{Antecedente de reacción adversa con productos con látex}

En 11 pacientes se encontró antecedente de reacción adversa con productos con látex ( $p=0.003$ ). En este grupo de pacientes las manifestaciones respiratorias fueron significativas $(p=0.012)$. No así las manifestaciones dermatológicas $(\mathrm{p}=0.548)$.

Cuadro 2. Relación de antecedente de atopia y prueba de PRICK positivo a látex

\begin{tabular}{lcc}
\hline Variable & $\mathbf{n ~ ( 1 6 )}$ & $\mathbf{p}$ \\
-Antecedente de atopia & $10(62 \%)$ & 0.047 \\
- Rinitis & 0 & 0.234 \\
- Asma & 0 & $\mathrm{~N}$ \\
- Dermatitis Atópica & $3(19 \%)$ & 0.657 \\
- Urticaria y Angioedema & $4(25 \%)$ & 0.043 \\
- Anafilaxia & $3(19 \%)$ & 0.008
\end{tabular}

Cuadro 3. Relación de reacción cruzada frutas-látex y alimentos asociados

\begin{tabular}{|c|c|c|}
\hline Variable & n (16) & p \\
\hline Reacción cruzada frutas-látex & $4(25 \%)$ & 0.043 \\
\hline \multicolumn{3}{|l|}{ Alimentos: } \\
\hline - Aguacate & $1(6 \%)$ & 0.130 \\
\hline - Papaya & $1(6 \%)$ & 0.130 \\
\hline - Fresa & $1(6 \%)$ & 0.130 \\
\hline - Kiwi & $1(6 \%)$ & 0.130 \\
\hline
\end{tabular}


Los productos con látex con los que hubo reacción fueron globos $(p=0.000)$ y guantes $(p=0.002)$. (Cuadro 4).

\section{Concentraciones séricas de IgE total}

La concentración de IgE total según los límites establecidos para edad fue normal en 14 pacientes, $y$ en dos estuvieron elevadas ( $p=0.643)$. La media reportada fue de $393 \mathrm{Ul} / \mathrm{mL}$. En el Cuadro 5 se resumen los factores clínicos asociados con las prueba de prick positivas al látex.

\section{DISCUSIÓN}

La alergia al látex es un problema de salud pública en pacientes de grupos de riesgo. El alar-

Cuadro 4. Antecedente de reacción adversa con productos con látex, manifestaciones clínicas y productos asociados

\begin{tabular}{lll}
\hline Variable & n (16) & p \\
-Reacción adversa con productos & $11(69 \%)$ & 0.003 \\
con látex & & \\
- Manifestaciones respiratorias n (\%) & $4(25 \%)$ & 0.012 \\
- Manifestaciones dermatológicas n (\%) & $1(7 \%)$ & 0.548 \\
- Anafilaxia n (\%) & $6(37 \%)$ & 0.000 \\
-Productos & & \\
- Globos n (\%) & $5(31 \%)$ & 0.000 \\
- Guantes n (\%) & $4(25 \%)$ & 0.002 \\
- Jeringas n (\%) & $1(7 \%)$ & 0.548 \\
- Sondas vesicales n (\%) & $1(7 \%)$ & 0.548
\end{tabular}

Cuadro 5. Factores clínicos asociados a prueba de PRICK positivo a látex

\begin{tabular}{lcc}
\hline Variable & OR & IC \\
-Manifestaciones respiratorias al & 11.66 & $(1.18-114.90)$ \\
contacto con productos con látex & & \\
-Manifestaciones dermatológi- & 2.33 & $(0.13-39.82)$ \\
cas al contacto con productos & & \\
con látex & & \\
-Antecedente de reacción adver- & & \\
sa con productos con látex & 1.02 & $(1.77-58.9)$ \\
- Globos & - & - \\
- Guantes & 2.4 & - \\
- Jeringas & 2.4 & $(0.140-$ \\
- Sondas vesicales & & $40.93)$ \\
& & $(0.140-40.93)$
\end{tabular}

OR: odds ratio, IC: Intervalo de confianza mante aumento de las reacciones anafilácticas al látex durante las cirugías o procedimientos radiológicos llevó a la Administración de Alimentos y Fármacos de los Estados Unidos (FDA) a publicar una serie de recomendaciones del tema en $1990 .{ }^{9}$

La prevalencia de sensibilización o alergia al látex en niños con espina bífida o con anomalías urogenitales varía entre $32.6 \%$ en estudios que utilizan pruebas cutáneas y $34-72 \%$ en los basados en pruebas serológicas. ${ }^{10}$

Esta prevalencia varía dependiendo del país, la metodología utilizada y las pruebas para establecer el diagnóstico; van de 6 a $60 \%$ en los niños con espina bífida. ${ }^{11}$

Nosotros encontramos una prevalencia de sensibilización al látex en pacientes con anomalías genitourinarias determinada mediante prueba cutánea de prick con extracto de látex de 30.7\%, en los pacientes pertenecientes al grupo de riesgo de niños con malformaciones genitourinarias y espina bífida.

Los principales factores de riesgo reportados en la bibliografía para la alergia o sensibilización a látex son los antecedentes atópicos y el número de intervenciones quirúrgicas. ${ }^{6,12,13}$

Se reporta que más de 5 o 6 cirugías constituyen un factor determinante para alergia al látex. ${ }^{14} \mathrm{Se}$ propone que la propia enfermedad podría constituir un factor específico en sí mismo, aunque existen posibles factores asociados que pueden ser determinantes a la hora de estimular el inicio de una sensibilización al látex (válvula de derivación ventrículo peritoneal, precocidad de las intervenciones, tipo de éstas, etc.). ${ }^{15}$

Yassin y colaboradores reportaron, como factor de riesgo, 12.67eventos quirúrgicos $(p=0.001),{ }^{12}$ mientras que Pires y su grupo encontraron que 
más de cuatro cirugías implicaban un riesgo significativo para sensibilización al látex en pacientes con espina bífida $(p=0.0001) .{ }^{11}$

En nuestro estudio la media de cirugías en pacientes con sensibilización al látex fue de 6.5. No se encontró asociación estadísticamente significativa ( $p$ 0.945) con sensibilización al látex, a diferencia de lo que la mayor parte de los estudios reporta ${ }^{12}$ de manera similar a lo informado por $\mathrm{H}$. Kattan en 59 pacientes con espina bífida de Arabia Saudita, donde tampoco se encontró que la cantidad de cirugías tuviera relación con la alergia al látex, porque el número de eventos quirúrgicos era bajo. ${ }^{13}$

La mayor parte de los estudios se efectuaron en pacientes con espina bífida, que son intervenidos quirúrgicamente desde edades muy tempranas- Además, existe una contribución genética potencial apoyada por la asociación del fenotipo HLA-DRB1_0701 (DR 7), con la respuesta mediada por IgE con el factor de elongación de goma Hev b1. Los fenotipos HLA-DRB4 y HLA-DQB8 se han relacionado con la producción de anticuerpos IgE en pacientes con espina bífida. ${ }^{14}$

El riesgo de alergia al látex es mayor cuando los individuos han sido intervenidos quirúrgicamente en los primeros días de vida. ${ }^{6}$ Pires y colaboradores, quienes estudiaron los factores de riesgo de sensibilización al látex en 57 pacientes con espina bífida encontraron que las cirugías en los primeros tres meses de vida fueron significativas como factor de riesgo para sensibilización al látex en pacientes con esa enfermedad ( $p=$ $0.008 ; \mathrm{RM}=5.4 ; \mathrm{IC} 95 \%=0.7-29.2) .{ }^{11}$

En nuestro estudio 11/16 pacientes con prueba de prick positiva al látex tuvieron su primera intervención quirúrgica antes del mes de edad; sin embargo, no encontraron asociación estadísticamente significativa $(p=0.209)$.
En el estudio de T Eiwegger, llevado a cabo en tres diferentes centros de Austria de 1996 a 2001, analizaron la exposición temprana a los productos del látex asociados con la sensibilización al mismo en tres diferentes grupos de pacientes: el grupo uno (pacientes con espina bífida), grupo dos (pacientes con hemorragia o hidrocefalia congénita), y grupo tres (pacientes con gastrosquisis y onfalocele). Encontraron que el primer grupo tuvo una prevalencia de $46 \%$ de sensibilización al látex $(\mathrm{p}=0.005)$ demostrada por IgE específica versus $8.9 \%$ de los pacientes del segundo grupo, y $5 \%$ en los del tercer grupo. Compararon también el número de cirugías como factor de riesgo para sensibilización al látex, que solo fue significativo en el grupo de pacientes con espina bífida $(p<0.05){ }^{16}$

En esta investigación se incluyeron pacientes con diferentes diagnósticos urológicos y no solo espina bífida o mielomeningocele, lo que pudiera explicar que el número de cirugías y edad a la primera intervención quirúrgica no tuvo relevancia significativa como lo demuestra T. Eiwegger en su estudio.

Se encontró que $62 \%$ de los pacientes con sensibilización al látex tenía antecedente de atopia $(p=0.047)$. Ausili y colaboradores reportaron el antecedente personal de atopia y sensibilización al látex en $62.5 \%$ de los pacientes con espina bífida $(p<0.01) .^{10}$

El hecho de ser atópico ejerce un efecto modulador en el sentido de que los pacientes atópicos parecen sensibilizarse con mayor rapidez y con menor número de intervenciones. Asimismo, la atopia se acompaña de mayor sensibilidad en los órganos diana, con un umbral más bajo para la aparición de síntomas clínicos que pueden producirse con concentraciones de IgE específica más bajas. ${ }^{15}$

Bueno de Sa y su grupo encontraron que $43.6 \%$ de los pacientes eran atópicos $(p=0.007) .{ }^{18}$ 
Yassin evaluó la alergia al látex en 76 pacientes con mielomeningocele y reportó el antecedente personal de atopia en $41 \%$ de los pacientes con alergia al látex $(\mathrm{p}=0.041) .^{12}$

En este estudio se encontró asociación entre el antecedente de reacción previa al contacto con productos con látex y prueba cutánea positiva al extracto de látex $(\mathrm{p}=0.003)$. En el estudio de Yassin también se reportó asociación entre la reacción previa con látex $(p<0.001)$. Ausili reportó que $37 \%$ de los pacientes de su estudio considerados como sensibilizados al látex tuvieron manifestaciones clínicas durante el contacto con el látex; sin embargo, no reporta los valores estadísticos de esta variable. ${ }^{10}$

El antecedente de manifestaciones respiratorias al contacto con productos con látex ocurrió en cuatro pacientes $(p=0.012)$, no se encontró relación con el antecedente de manifestaciones dermatológicas con látex $(p=0.548)$.

Se reporta que 4 de los 16 pacientes con sensibilización al látex tenían antecedente de reacción al contacto con productos con látex, sobre todo con globos y guantes $(\mathrm{p}=0.002 \%)$, los otros productos, como guantes, sondas, jeringas, no se asociaron con sensibilización al látex. En el estudio de T. Michael 14 de 165 pacientes estudiados con espina bífida tenían antecedente de síntomas clínicos al inflar globos lo que se asoció con sensibilización al látex. ${ }^{19}$

El $25 \%$ de los pacientes tuvo reacción cruzada al látex-frutas determinada mediante interrogatorio $(p=0.043)(R M: 5.6$ IC: $0.91-34)$, los alimentos fueron: aguacate, papaya, kiwi, fresa. Los pacientes refirieron síntomas de síndrome de alergia oral en los cuatro casos. Diversos trabajos han demostrado que entre 20 y $60 \%$ de los pacientes alérgicos al látex experimentan reacciones mediadas por IgE a una amplia variedad de alimentos, sobre todo frutas como: plátano, aguacate, castaña y kiwi. ${ }^{20}$
En las concentraciones de laboratorio de IgE sérica total no se encontró asociación significativa. La mayor parte de los estudios reporta cifras elevadas de IgE específica para látex asociadas con alergia o sensibilización al látex; sin embargo, no existen muchos reportes que describan asociación de IgE sérica total con sensibilización al látex. En el estudio de Pires se encontró asociación estadísticamente significativa con concentraciones de IgE sérica total $\geq 44 \mathrm{IU} /$ $\mathrm{mL}(\mathrm{p}=0.02 ; \mathrm{RM}=8.6 ; 95 \% \mathrm{Cl}=1.4-53.4)$. En comparación con nuestra investigación, no encontramos asociación con concentraciones elevadas de IgE sérica total con sensibilización al látex $(\mathrm{p}=0.883$ ) aunque se reportó una mediana de $57.250 \mathrm{IU} / \mathrm{mL}$ se tomaron las concentraciones de IgE según los límites establecidos para edad, donde el límite superior normal para las edades de 6-8 años fue de $161.3 \mathrm{IU} / \mathrm{mL}$ y de 9 a 10 años límite superior normal de $570.6 \mathrm{IU} / \mathrm{mL} .{ }^{21}$

El diagnóstico oportuno de sensibilización al látex en pacientes de grupos de riesgo permite implementar las medidas de prevención de alergia al látex y, de esta manera, disminuir el riesgo de reacciones severas, lo que disminuirá la morbilidad y mortalidad y los costos de atención médica. Es importante conocer la prevalencia de alergia al látex en nuestro país en el grupo de pacientes de riesgo, y realizar la prueba de prick a látex para determinar en quiénes deberá utilizarse quirófano libre de látex. El reconocimiento de este problema permitirá establecer las medidas de prevención primaria que han permitido en los países industrializados un descenso de la prevalencia de alergia al látex.

\section{Agradecimientos y declaraciones}

Agradecemos al equipo del laboratorio del Hospital de Pediatría del Centro Médico Nacional de Occidente el apoyo para el procesamiento de IgE sérica total. Al doctor Julio César Gómez Castellanos por el apoyo en el envío de pacien- 
tes con factores de riesgo para la realización de prueba de prick al látex.

\section{REFERENCIAS}

1. Slater JE. Latex allergy. En: Kay AB, ed. Allergy and allergic diseases. Oxford: Blackwell Science: 1997;981-93.

2. Cabañes N. Latex Allergy: Position Paper. J Invest Allergol Clin Immunol. 2012;22(5):313-330

3. Stern G. Uberempfindlichkeit gegen kaustchuk als urasche von Urticaria and quinckeschemodem. Klin Wochenschrift. 1927;6:1096-7.

4. Nutter AF. Contact urticaria to rubber. Br J Dermatol. 1979;101:597-8.

5. Kahn SL, Podjasek JO, Dimitropoulos VA, Brown CW Jr. Natural rubber latex allergy. Dis Mon. 2016;62(1):5-17.

6. Liss GM, Sussman GL. Latex sensitization: occupational versus general population prevalence rates. Am J Ind Med. 1999;35:196-200.

7. Negro Alvarez JM, Miralles López JC. Alergia al látex. Rev Clin Esp. 2003;34;2;28-35.

8. Blanco C. Latex-fruit syndrome. Curr Allergy Asthma Rep 2003;3:47-53.

9. Thompson RL. Educational challenges of latex protein allergy. Immunol Allergy Clin North Am. 1995;15:159-74.

10. Ausili E, Tabacco F, Focarelli B, Nucera E, Patriarca G, Rendeli C. Prevalence of latex allergy in spina bifida: genetic and environmental risk factors. Eur Rev Med Pharmacol Sci. 2007;11(3):149-153.

11. Pires G, Morais-Almeida M, Gaspar A, Godinho N, Calado $E$, Abreu-Nogueira J. Risk factors for latex sensitization in children with spina bifida. Allergol et Immunopathol. 2002;30(1):5-13

12. Yassin MS, Sanyurah, Fischer TJ. Evaluation of latex allergy in patients with meningomyelocele. Ann Allergy 1992;69(3):207-11 .

13. Kattan H., Har H.A., Tipirneni P. Latex allergy in Saudi children with spina bifida. Allergy. 1999;54:70-3.

14. Buck D, Michael T, Wahn U, Niggemann B. Ventricular shunts and the prevalence of sensitization and clinically relevant allergy to latex in patients with spina bifida. Pediatr Allergy Immunol. 2000;11:111-5.

15. Kelly KJ, Pearson ML, Kurup VP, Havens PL, Byrd RS, Setlock, et al. Acluster of anaphylactic reactions in children with spina bifida during general anesthesia: epidemiologic features, risk factors and latex hypersensitivity. J Allergy Clin Immunol. 1994;94(1):53-61.

16. Eiwegger T, Dehlink E, Schwindt J, Pomberger G, Reider N, Frigo E, Rokitansky AM, Urbanek R, Szépfalusi Z. Early exposure to latex products mediates latex sensitization in spina bifida but not in other diseases with comparable latex exposure rates. Clin Exp Allergy. 2006;36(10):1242-6.

17. Turjanmaa K. Diagnosis of latex allergy. Allergy. 2001;56:810-2.

18. Bueno de Sá A, Faria Camilo Araujo, Cavalheiro S, Carvalho Mallozi. Profile of latex sensitization and allergies in children and adolescents with myelomeningocele in Sao Paulo, Brazil. J Invest Allergol Clin Immunol. 2013;23(1):43-49.

19. Michael T, Niggemann B, Moers A, Seidel U. Risk factors for latex allergy in patients with spina bifida. Clinical and Experimental Allergy, 1996;26:934-9.

20. García JC, Moyano JC, Álvarez M, Bellido J. Latex allergy in fruit-allergic patients. Allergy. 1998;53:532-6

21. Castor J. Rau R, The Harriet Lane Handbook. 19 ed. MosbyElsevier, 2012;395. 